\title{
Trastuzumab in combination with AT-101 induces cytotoxicity and apoptosis in Her2 positive breast cancer cells
}

\author{
Gulcan Bulut*,1 iD, Harika Atmaca ${ }^{2}$ \& Burcak Karaca ${ }^{1}$ \\ ${ }^{1}$ Division of Medical Oncology, Tulay Aktas Oncology Hospital, School of Medicine, Ege University, 35100, Bornova, Izmir, Turkey \\ ${ }^{2}$ Section of Molecular Biology, Department of Biology, Faculty of Science \& Letters, Celal Bayar University, 45140, Muradiye, \\ Manisa, Turkey \\ *Author for correspondence: Tel.: +90 232390 4387; Fax: + 90232374 7321; gulcanbulut07@gmail.com
}

\begin{abstract}
Aim: AT-101 is a polyphenolic compound with potent anti-apoptotic effects in various cancers. In this study, the possible synergistic cytotoxic and apoptotic effect of trastuzumab/AT-101 combination was investigated in HER2-positive breast cancer cell lines. Materials \& methods: SKBR-3, MDA-MB-453 and MCF-10A cell lines were treated with a trastuzumab/AT-101 combination. Synergistic cytotoxicity and apoptosis effects were shown and then PI3K and Akt protein levels were studied. Result: The trastuzumab/AT-101 combination induced synergistic cytotoxicity and apoptosis in both breast cancer cells but not in MCF10A cells. Combination treatment induced cytotoxicity via inhibiting PI3K/AKT but not the MAPK/ERK pathway. Conclusion: The trastuzumab/AT-101 combination may be a good candidate for patients with trastuzumab-resistant Her2-positive breast cancer and inhibition of the PI3K/AKT pathway may be one of the underlying mechanisms.
\end{abstract}

First draft submitted: 4 September 2019; Accepted for publication: 27 November 2019; Published online: 12 December 2019

Keywords: apoptosis • AT-101 • PI3K/AKT • synergism • trastuzumab

Breast cancer is one of the most common cancers among women, and is the second leading cause of cancer deaths [1]. It is a highly heterogeneous disease with different clinical outcomes. The HER2 oncogene is over expressed in $25-30 \%$ of all breast cancer cases, and over expression of this oncogene is closely related to shortened diseasefree survival [2,3]. Treatment of these cancers is based on targeted drugs or combinations because HER2-positive breast cancers are generally estrogen receptor (ER)-negative. Trastuzumab is a monoclonal targeted agent against HER2. HER2-positive patients who are treated with trastuzumab have good clinical responses. However, 50\% of the patients are resistant to trastuzumab either primary or secondary. The activation of PI3K signal transduction pathway may be one of the trastuzumab resistance mechanisms [2-5].

Gossypol is a polyphenolic compound found in the seeds of the cotton plant and Thespesia populnea [5]. AT-101(R(-)-1,1', 6,6',7,7'-hexahydroxy-3,3'-dimethyl-5,5'-bis (1-methylethyl) [2,2'-Binapthalene]-8, 8'-dicarboxaldehyde acetic acid), which is the (-) enantiomer of gossypol has been found to have a more potent cytotoxic effect than the $(+)$ enantiomer or racemic gossypol. In vitro and in vivo antiproliferative and apoptotic effects of AT-101 have been shown in various cancers such as breast, ovarian, uterine, adrenal, pancreatic, head and neck [6-8]. Moreover, through clinical studies, it has been shown that AT-101 is less toxic and well tolerated by patients, and thereby it is considered as a potential agent for combination therapies [9-12].

The cell culture assay was set up using two breast cancer cell lines with HER2 overexpression. These breast cancer cell lines were MDAMB453 and SKBR-3. The MDA-MB453 cell line is aneuploid human female with chromosome counts in the hypo-to-near-tetraploid range. This cell line was derived in 1976 by Cailleu et al. from an effusion of a 48-year-old female patient with metastatic breast cancer involving the nodes, brain and both pleural and pericardial effusion. FGF and HER2 are expressed by these cells. The SKBR-3 cell line is a hypertriploid line with a modal chromosome number of $84,34 \%$ of these cells have 80 chromosomes as well as a high rate (28\%) of ploidy. $35-40 \%$ of chromosomes in a cell complemented with a modal chromosome number of 84 consisted of 
structurally altered marker chromosomes. This cell line was derived by G Trempe and LJ Old in 1970 from pleural effusion cells of a patient, a white, Caucasian female, 43 years of age with blood type A+, who had been treated with radiation, steroids, cytoxan and 5-fluorouracil. This cell line is overexpressed HER2.

In this study, we investigated the possible synergistic cytotoxic effect of a trastuzumab/AT-101 combination in SKBR-3 and MDA-MB-453 HER2-positive breast cancer cell lines. To evaluate the action mechanisms of the combination treatment, we investigated the effect of the combination treatment on PI3K/AKT and MAPK/ERK signal transduction pathways, and further evaluated the apoptotic activity of this combination treatment.

\section{Materials \& methods}

Cell lines \& reagents

SKBR-3 and MDA-MB-453, which are human breast cancer HER2-positive cell lines were obtained from Interlab Cell Line Collection (ICLC, Genova, Italy), and MCF-10A cells were purchased from Health Protection Agency (HPA, London, UK). The cells were grown as monolayer adherent cell lines and were routinely cultured in Dulbecco's modified Eagle medium/nutrient F-12 Ham supplemented with 10\% heat-inactivated fetal bovine serum, $1 \%$ L-glutamine, $1 \%$ penicillin-streptomycin in $75 \mathrm{~cm}^{2}$ polystyrene flasks (Greiner Bio-One GmbH, Kremsmuenster, Austria), and maintained at $37^{\circ} \mathrm{C}$ in a humidified atmosphere with $5 \% \mathrm{CO}_{2}$. Cell culture supplies were obtained from Biological Industries (Merck Millipore, Darmstadt, Germany).

AT-101 was received from Ascenta Therapeutics (PA, USA). Trastuzumab was obtained from Roche Applied Science (Mannheim, Germany). The stock solutions of AT-101 $(10 \mu \mathrm{M})$ and trastuzumab $(10 \mathrm{mg} / \mathrm{ml})$ were formulated in DMSO. They were saved at $-20^{\circ} \mathrm{C}$. The dilutions which were prepared were made just before we use them, and we made new stock solutions for each experiment we did. In the study, the DMSO concentration did not exceed $0.1 \%$ and even in this condition, it was not cytotoxic to the cells. All other chemicals which were not cited above were purchased from Sigma, Louis, Missouri, ABD (with the method recommended by Sigma).

\section{$\mathrm{XTT}$ viability assay}

Cells were seeded at nearly $1 \times 10^{4}$ cells/well in a final volume of $100 \mu \mathrm{l}$ in 96-well flat-bottom microtiter plates. The cells were treated various concentrations of trastuzumab and AT-101 alone or in combination after an overnight incubation. Plates were incubated at $37^{\circ} \mathrm{C}$ in a $5 \% \mathrm{CO}_{2}$ incubator for 24,48 and $72 \mathrm{~h}$. We did not stimulate media throughout this period. At the end of incubation, we added $100 \mu \mathrm{l}$ of XTT (Roche Applied Science) to each well, and after that we incubated the plates at $37^{\circ} \mathrm{C}$ for another $4 \mathrm{~h}$. Later, absorbance was measured by a microplate reader (DTX 880 Multimode Reader, Beckman Coulter, FL, USA) from 450 to $650 \mathrm{~nm}$ wavelength. In order to calculate the $\mathrm{IC}_{50}$ values, we used the mean of triplicate experiments for each dose combination index $(\mathrm{CI})$ values were calculated using the CalcuSyn software.

\section{DNA fragmentation analysis}

Apoptosis was evaluated using ELISA using the Cell Death Detection ELISA ${ }^{\text {Plus }}$ Kit (Roche Applied Science). We measured the related amounts of mono- and oligonucleosomes originated from the apoptotic cells by monoclonal antibodies directed against DNA and histones having ELISA. Absorbance was measured at $405 \mathrm{~nm}$ with a reference wavelength at $490 \mathrm{~nm}$ (DTX 880 Multimode Reader, Beckman Coulter). The cytoplasmic fraction of the untreated control and treated cells were sent onto a streptavidin-coated plate, and it was incubated with a mixture of biotin labeled antihistone and peroxidase conjugated anti-DNA for $2 \mathrm{~h}$ at room temperature. The plate was washed completely and incubated with 2,29-azino-di-[3-ethylbenzthiazolinesulphonate] diammonium salt, then absorbance was quantified at $405 \mathrm{~nm}$ with a reference wavelength at $490 \mathrm{~nm}$ (DTX 880 Multimode Reader, Beckman Coulter). We did all experiments as triplicate.

\section{Caspase $3 / 7$ assay}

Apoptosis was verified by using the caspase-Glo 3/7 Assay (Promega, WI, USA) according to the manufacturer's instructions. Briefly, cells were placed in a 96-well plate in $100 \mu \mathrm{l}$ culture medium in the absence or presence of increasing concentrations of drugs alone or in combination. After that, $100 \mu \mathrm{l}$ of caspase-Glo 3/7 reagent was transferred onto each well and the plates were incubated for $1 \mathrm{~h}$ at room temperature. At the end of the incubation, the luminescence of each sample was measured in a luminometer (DTX 880 Multimode Reader, Beckman Coulter). 


\section{Western blot analysis}

The M-PER Mammalian Protein Extraction Reagent (Thermo Scientific, IL, USA) was used to extract total protein. Total protein concentrations were determined using Bradford assay (Bio-Rad Laboratories, CA, USA). Equal amounts of proteins along with molecular weight marker were loaded onto SDS polyacrylamide gel and separated using electrophoresis (PAGE). Gels were run for approximately $2 \mathrm{~h}$ at $120 \mathrm{~V}$ for PAGE. After PAGE, proteins were transferred from the gels into nitrocellulose membranes (Bio-Rad Laboratories) by running the sandwich for $75 \mathrm{~min}$ at $115 \mathrm{~V}$, at $4^{\circ} \mathrm{C}$. To verify the transfer step, the membranes and gels were stained with Ponceau S (Sigma Chemical Co., MO, USA) and Coomassie Brilliant Blue R-250 (Bio-Rad Laboratories), respectively. The membranes were blocked using blocking buffer prepared with 5\% nonfat dry milk (Bio-Rad Laboratories) in tris-buffered saline containing $0.1 \%$ Tween 20 for $1 \mathrm{~h}$, at room temperature. Then, the membranes were incubated with primary antibodies, anti-MAPK (Abcam, ab185145), anti-Pi3k (Abcam, ab32089), anti-beta actin antibody (Abcam, ab8226), anti-ERK1 antibody (Abcam, ab32537), anti-akt antibody (Abcam, ab32505), which were obtained from Abcam, for $16 \mathrm{~h}$, at $4^{\circ} \mathrm{C}$. After several washing steps with tris-buffered saline containing $0.1 \%$ Tween 20, membranes were incubated with secondary antibodies for $1 \mathrm{~h}$, at room temperature. Protein bands were visualized using the Kodak Gel Logic 1500 Imaging System (Carestream Molecular Imaging, Newhaven, CT, USA). All antibodies, unless mentioned, were purchased from Abcam (Cambridge, UK). $\beta$-Actin was evaluated as a loading control and all incubation steps were performed in orbital shaker.

\section{Statistical analysis}

All experiments were carried out in triplicate and the results are stated as the mean \pm standard deviation. The data were analyzed through one-way analysis of variance followed by Dunnett's t-test for multiple comparisons. Values with $\mathrm{p}<0.05$ were regarded as statistically important. We calculated determination of the half maximal (50\%) inhibitory concentrations $\left(\mathrm{IC}_{50}\right.$ ) of each agent and the synergistic cytotoxic effects of cells treated with trastuzumab and AT-101 combination by the BiosoftCalcuSyn 2.1 software (MO, USA). We used the CI to state additive effect $(\mathrm{CI}=1)$, antagonism $(\mathrm{CI}>1)$, synergism $(\mathrm{CI}<1)$ and strong synergism $(\mathrm{CI}<0.5)$ [13]. Data analysis and graph production were achieved using the GraphPad Prism 6.0 software (CA, USA; similar to the method in the article presented by Chou et al.).

\section{Results}

Trastuzumab in combination with AT-101 induces synergistic cytotoxicity in human HER2+ breast cancer cells but not in MCF-10A cells

To evaluate the effects of trastuzumab and AT-101 on the viability of SKBR-3 and MDA-MB-453 human breast cancer cells and MCF-10A cells, cells were exposed to increasing concentrations of trastuzumab $(1-100 \mu \mathrm{g} / \mathrm{ml})$ or AT-101 $(2.5-15 \mu \mathrm{M})$ for 24, 48 and $72 \mathrm{~h}$, and an XTT cell viability assay was performed. Both trastuzumab and AT-101 decreased cell viability in a time- and dose-dependent manner in SKBR-3 and MDA-MB- 453 human breast cancer cells. The highest cytotoxicity was observed at $72 \mathrm{~h}$ (Figure $1 \mathrm{~A}-\mathrm{D}$ ) and $\mathrm{IC}_{50}$ values of trastuzumab in SKBR-3 and MDA-MB-453 cells were calculated as 17.6 and $97.9 \mu \mathrm{g} / \mathrm{ml}$, respectively. The $\mathrm{IC}_{50}$ of AT-101 was $6.3 \mu \mathrm{M}$ for SKBR-3 cells and $8.7 \mu \mathrm{M}$ for MDA-MB-453 cells, respectively.

To assess the possible synergistic effects of trastuzumab and AT-101 combination, SKBR-3 and MDA-MB-453 cells were exposed to different concentrations of the drugs alone, and combined for $72 \mathrm{~h}$. The results showed that $100 \mu \mathrm{g} / \mathrm{ml}$ trastuzumab and $2.5 \mu \mathrm{M}$ AT-101 resulted in a 42 and $19 \%$ decrease in proliferation of MDA-MB453cells, respectively, while both together at the same doses caused a $75 \%$ decrease in cell viability compared with untreated controls, indicating strong synergy ( $\mathrm{p}<0.05$; Figure $2 \mathrm{~A}$ ). There was significant synergistic cytotoxicity in SKBR-3 cells at $72 \mathrm{~h}$ compared with either agent alone; $10 \mu \mathrm{g} / \mathrm{ml}$ trastuzumab and $2.5 \mu \mathrm{M}$ AT-101 resulted in a 23 and 19\% decrease, respectively, in the viability of SKBR-3 cells. However, the combination resulted in a 71\% decrease in viability $(\mathrm{p}<0.05$; Figure $2 \mathrm{~B})$.

The concentrations for each agent that were found to be synergistic on SKBR-3 and MDA-MB-453 cells are given in Table 1 . However, neither $10 \mu \mathrm{g} / \mathrm{ml}$ trastuzumab and $2.5 \mu \mathrm{M}$ AT-101 nor $100 \mu \mathrm{g} / \mathrm{ml}$ trastuzumab and $2.5 \mu \mathrm{M}$ AT-101 treatments were cytotoxic to MCF-10A normal breast cells (data not shown). 


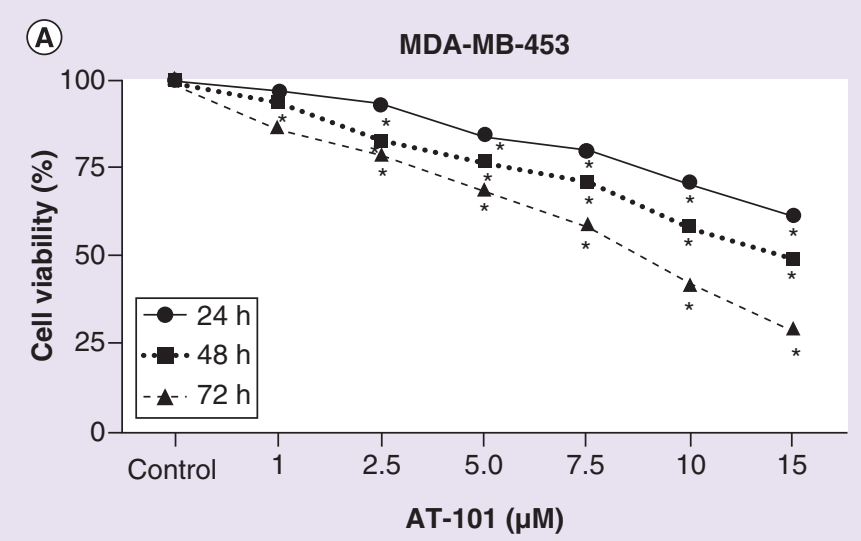

(C)

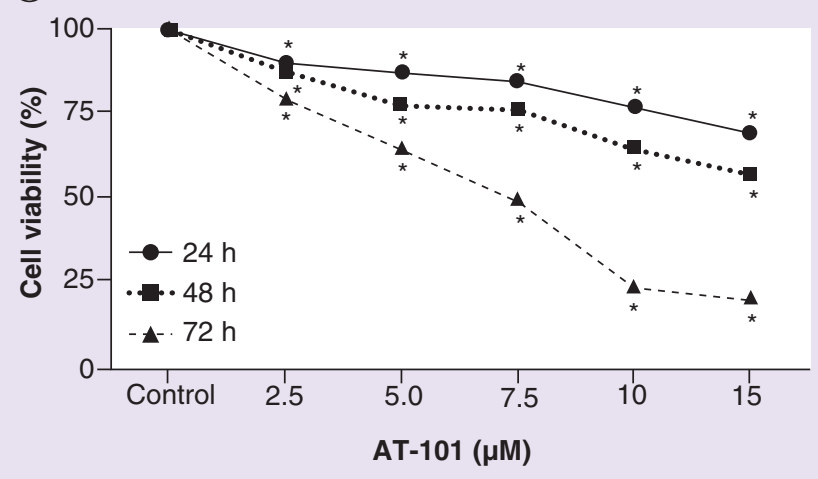

(B)

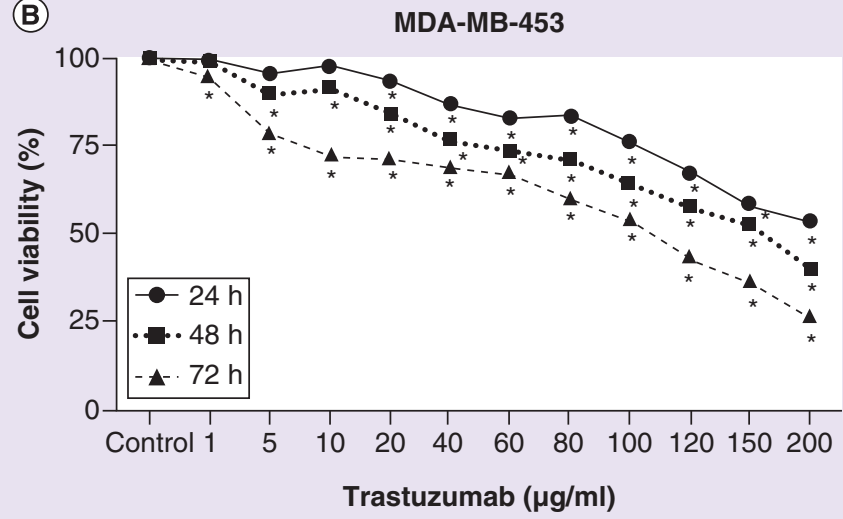

(D)

SKBR-3

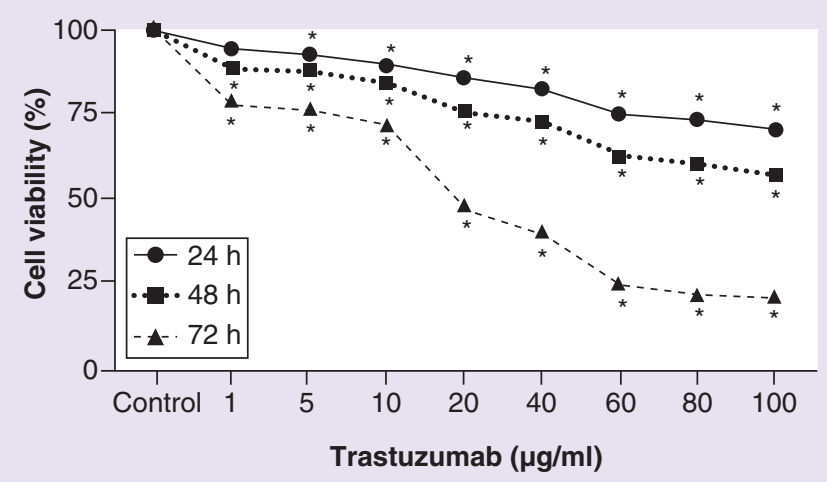

Figure 1. Concentration and time-dependent effects of trastuzumab and AT-101 on the viability of MDA-MB-453 and SKBR-3 cell lines. ${ }^{*} p<0.05$; significant as compared with the control group.

\begin{tabular}{|c|c|c|c|c|}
\hline & AT-101 $(\mu \mathrm{M})$ & Trastuzumab $(\mu \mathrm{g} / \mathrm{ml})$ & $\mathrm{Cl}$ & Interpretation \\
\hline \multirow[t]{3}{*}{ SKBR-3 } & 2.5 & 10 & 0.290 & Strong synergism \\
\hline & 5 & 5 & 0.508 & Synergism \\
\hline & 5 & 10 & 0.588 & Synergism \\
\hline \multirow[t]{2}{*}{ MDA-MB-453 } & 2.5 & 100 & 0.236 & Strong synergism \\
\hline & 5 & 80 & 0.373 & Strong synergism \\
\hline
\end{tabular}

Trastuzumab \& AT-101 combination induces cytotoxicity by inhibiting the PI3K/AKT pathway, but not the MAPK/ERK pathway

To explaining the underlying mechanisms of the synergistic apoptotic effects of the combination treatment, we investigated whether the cytotoxicity was related to inhibition via of the PI3K/AKT and/or MAPK/ERK signaling pathways. For this aim, the effects of a specific PI3K/AKT inhibitor (LY294002) or specific ERK inhibitor (FR180204) were investigated on the viability of breast cancer cells using an XTT viability assay. Breast cancer cells were pretreated with LY294002 for $1 \mathrm{~h}$ before treatment with the combination of $10 \mu \mathrm{g} / \mathrm{ml}$ trastuzumab 
(A)

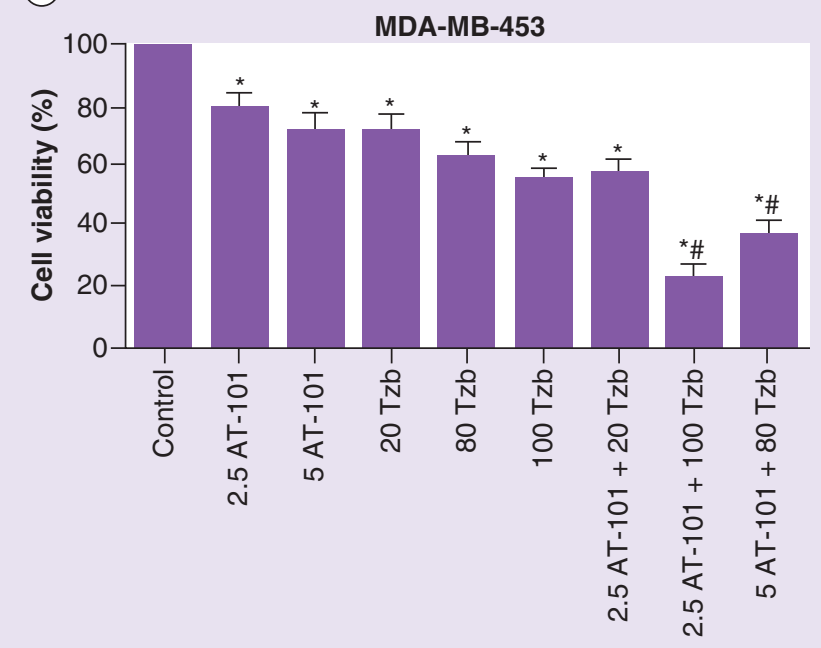

AT-101 $(\mu \mathrm{M})$, trastuzumab $(\mu \mathrm{g} / \mathrm{ml}), 72 \mathrm{~h}$
(B)

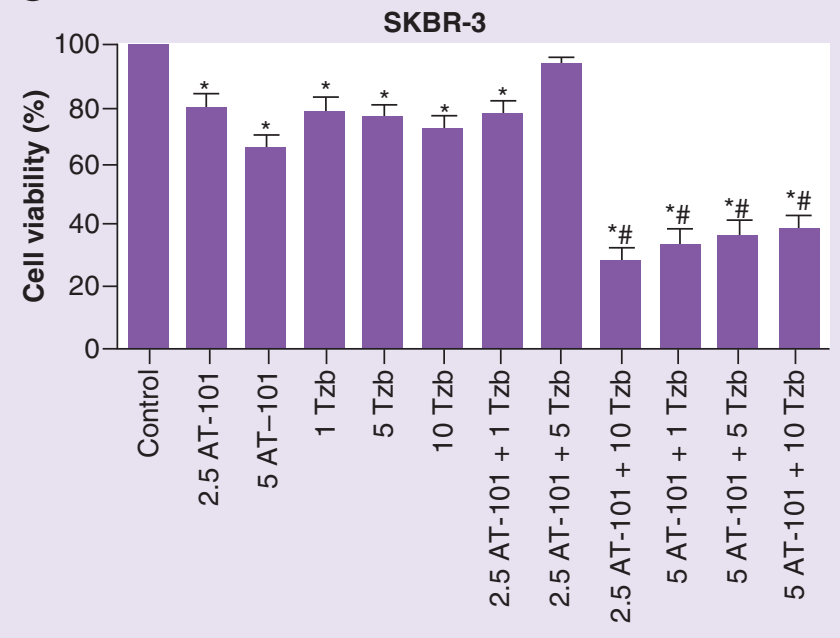

AT-101 $(\mu \mathrm{M})$, trastuzumab $(\mu \mathrm{g} / \mathrm{ml}), 72 \mathrm{~h}$

Figure 2. Effects of the trastuzumab/AT-101 combination on the viability of MDA-MB-453 and SKBR-3. (A \& B) Breast cancer cells at $72 \mathrm{~h}$. ${ }^{*} \mathrm{p}<0.05$; significant as compared with the control group; ${ }^{*} \mathrm{p}<0.05$; significant as compared with both the control and single-agent-treated groups.

and $2.5 \mu \mathrm{M}$ AT-101, and then the viability of cells was investigated. It was worked out that the viability of the combination-treated cells was not highly changed in the presence of LY294002 $(50 \mu \mathrm{M})$ as compared with nontreated cells (Figure 3A \& D; p < 0.05). Cells were pretreated with FR180204 for $1 \mathrm{~h}$ before treatment with the AT-101/trastuzumab combination, and then the viability of cells was investigated using the XTT assay. Cell viability was significantly decreased by the AT-101/trastuzumab combination as compared with FR180204-treated cells (Figure 3B-E; $\mathrm{p}<0.05$ ). Furthermore, PI3K and AKT protein levels were evaluated after the combination treatment using western blot analysis. Both $\mathrm{P} 3 \mathrm{~K}$ and AKT levels were inhibited by the combination treatment in SKBR-3 and MDA-MB-453 cells (Figure 3C-F).

\section{Combination of trastuzumab with AT-101 induces apoptosis in human HER2+ breast cancer cells}

To investigate the effect of the trastuzumab/AT-101 combination on the induction of apoptosis, SKBR-3 and MDA-MB-453 cells were treated with different concentrations of trastuzumab or AT-101 or both together for $72 \mathrm{~h}$ before analyzing the levels of mono-oligonucleosome fragments using Cell Death Detection ELISA ${ }^{\text {Plus }}$. Both agents induced apoptosis in a dose-dependent manner in SKBR-3 and MDA-MB-453 cells (Figure 4A-D).

When SKBR-3 cells were exposed to $100 \mu \mathrm{g} / \mathrm{ml}$ trastuzumab and $2.5 \mu \mathrm{M}$ AT-101, there was a 7.3- and 4.5-fold increase in DNA fragmentation, respectively. However, the combination induced a 14.2-fold increase in DNA fragmentation versus untreated controls $(\mathrm{p}<0.05$; Figure $5 \mathrm{~A})$. When MDA-MB- 453 cells were exposed to $100 \mu \mathrm{g} / \mathrm{ml}$ trastuzumab and $2.5 \mu \mathrm{M}$ AT-101, there was a 5.2- and 2.7-fold increase in DNA fragmentation, and the combination induced an 9.6-fold increase in DNA fragmentation versus untreated controls $(\mathrm{p}<0.05$; Figure 5B).

Moreover, to verify the induction of apoptosis by the combination treatment in breast cancer cells, we evaluated the activation of caspase 3/7 in both cells. In parallel with DNA fragmentation analysis, the combination treatment induced caspase 3/7 activity in both breast cancer cells as compared with single agents and untreated controls $(\mathrm{p}<0.05$; Figure 5C \& D). We also verified the induction of apoptosis by measuring DNA fragmentation after inhibiting caspase-8 or caspase-9. Cells were pretreated with a caspase-8 (z-IETD-fmk)-specific inhibitor, then exposed to the trastuzumab/AT-101 combination. As seen in Figure 6, there was a decrease in apoptosis as compared with combination-treated cells $(\mathrm{p}<0.05)$. Similar results were obtained by caspase 9 (z-LEHD-fmk) pretreatment in both breast cancer cells (Figure 6A \& B), indicating that the combination treatment activated both the extrinsic and intrinsic pathways of apoptosis in SKBR-3 and MDA-MB-453 cells. 


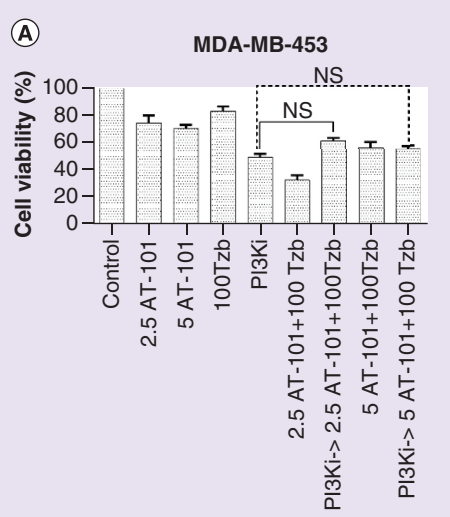

AT-101 ( $\mu \mathrm{M})$, trastuzumab $(\mu \mathrm{g} / \mathrm{ml}), 72 \mathrm{~h}$

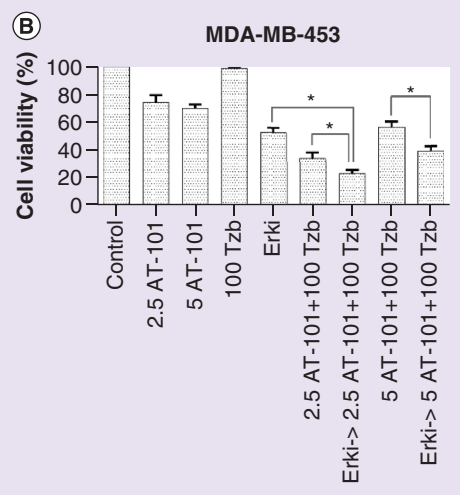

AT-101 $(\mu \mathrm{M})$, trastuzumab $(\mu \mathrm{g} / \mathrm{ml}), 72 \mathrm{~h}$

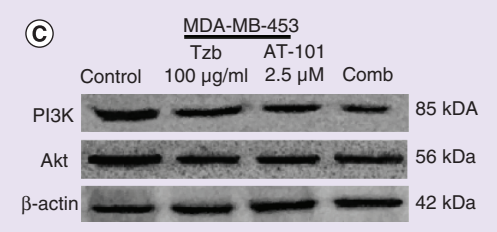

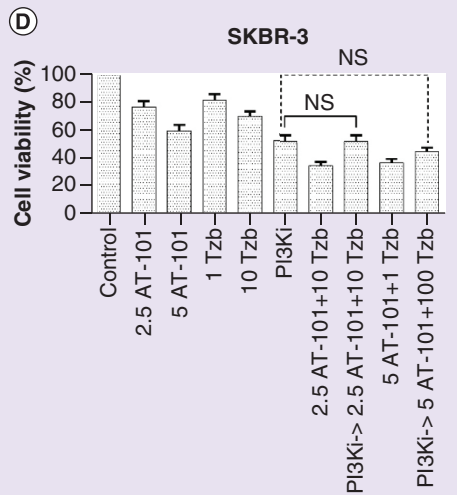

AT-101 ( $\mu \mathrm{M})$, trastuzumab $(\mu \mathrm{g} / \mathrm{ml}), 72 \mathrm{~h}$
(E)

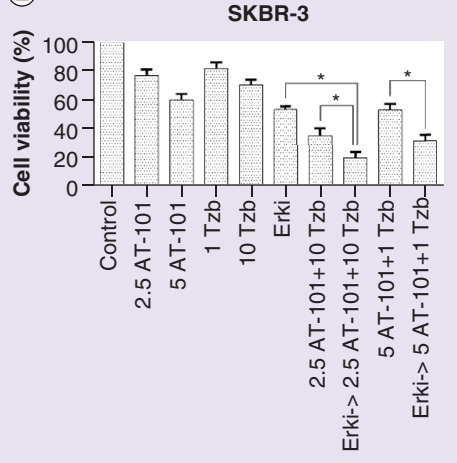

AT-101 $(\mu \mathrm{M})$, trastuzumab $(\mu \mathrm{g} / \mathrm{ml}), 72 \mathrm{~h}$

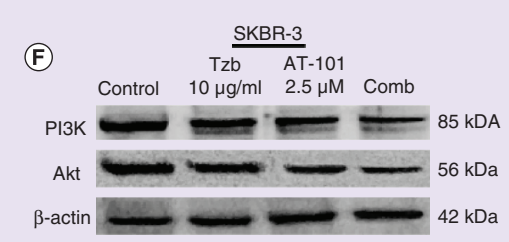

Figure 3. Effects of (A-D) LY294002 and (B-E) FR180204 on the viability of the trastuzumab/AT-101 combination-treated MDA-MB-453 and SKBR-3 cells. Western blot analysis of PI3K and Akt proteins after treatment with the trastuzumab/AT-101 combination in (C) MDA-MB-453 and (F) SKBR-3 breast cancer cells.

${ }^{*} \mathrm{p}<0.05$; significant as compared with the control group; ${ }^{*} \mathrm{p}<0.05$; significant as compared with both the control and single-agent-treated groups.

\section{Discussion}

Trastuzumab is a humanized monoclonal antibody targeted against the HER2/neu receptors [5,14]. In early stage HER2-positive breast cancer, trastuzumab-containing regimens improve overall survival and disease-free survival; however, patients who initially respond to trastuzumab develop resistance within 1 year of treatment [14]. Therefore, novel combination treatments are needed for patients with HER2-positive breast cancer.

AT-101 mimics BH3-only proteins, induces production of reactive oxygen species, and thus triggers apoptosis of cancer cells [5]. The BH3-only proteins of the Bcl-2 family (having only the Bcl-2 homology domain $\mathrm{BH} 3$ ) can trigger apoptosis by binding to proapoptotic members of this family and neutralizing their functional activity. AT-101 binds to the BH3-binding pocket of $\mathrm{Bcl}-\mathrm{xl}$ and $\mathrm{Bcl}-2$ and disrupts the heterodimerization of $\mathrm{Bcl}-2$ with the proapoptotic family members myeloid cell leukemia 1 and Bcl-xl. Although the effect of AT-101 on basic apoptotic genes such as Bcl-2 and bax is known, the activity of other apoptotic genes in AT-101 activity must be investigated. This is a strong inducer of apoptosis in various cancer cells and is now in phase II clinical trials for hormone-refractory prostate, breast and lung cancer, and leukemia [6-8]. Due to its acceptable adverse effects, it is considered as a potential agent for combination therapies.

In this study, we investigated the possible synergistic cytotoxic effects of trastuzumab in combination with AT-101 in HER2-positive breast cancer cells and found that the combination treatment resulted in synergistic cytotoxicity as compared with any single agent alone. Due to the differences in their genetic properties, SKBR-3 and MDA- 
(A)

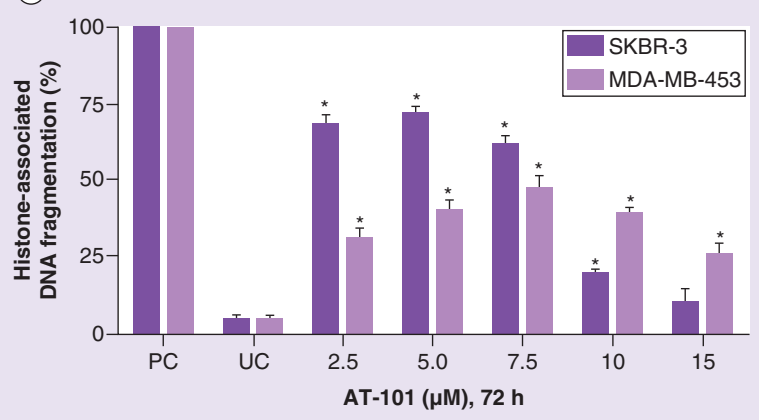

(C)

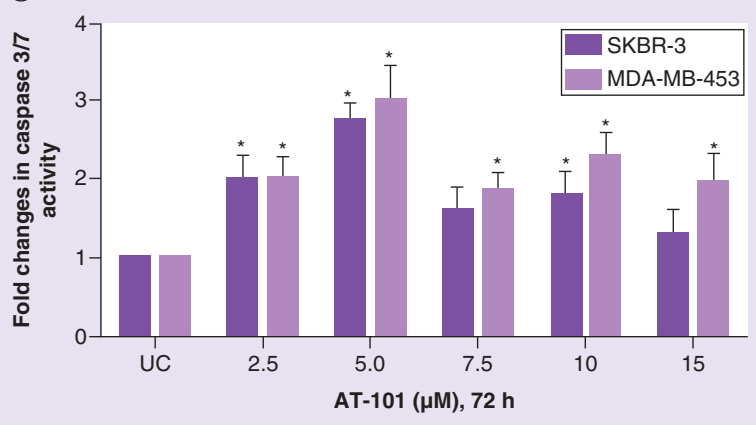

(B)

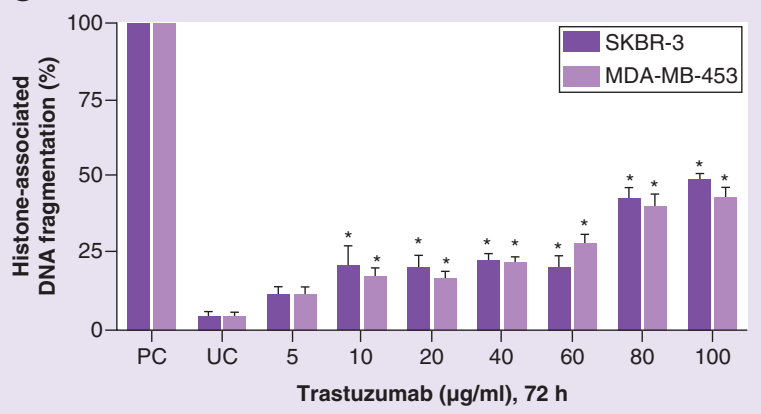

(D)

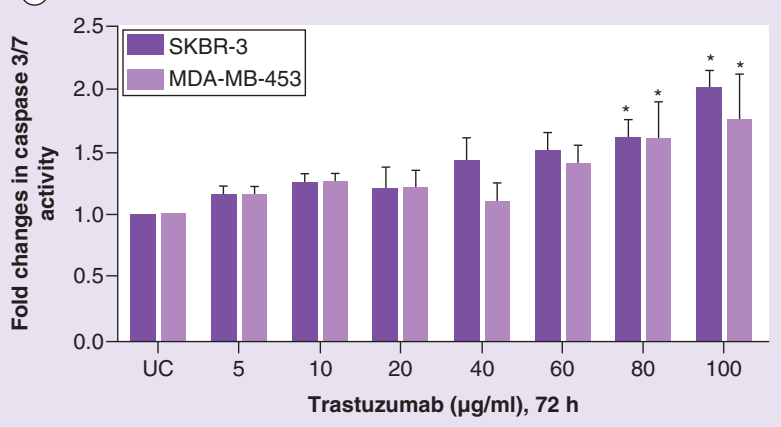

Figure 4. Concentration-dependent analysis of apoptosis through (A \& B) DNA fragmentation and (C \& D) caspase 3/7 activity assays in trastuzumab or AT-101 treated MDA-MB-453 and SKBR-3 cells at $72 \mathrm{~h}$.

${ }^{*} \mathrm{p}<0.05$; significant as compared with the control group.

PC: Positive control; UC: Untreated control.

MB-453 cells responded differently to the effects of the combination treatment, suggesting that MDA-MB-453 cells were much more resistant. However, the combination treatment showed less cytotoxicity against MCF-10A cells,indicating that the combination treatment might be tumor specific.

Although searching for the underlying mechanism of the synergism of trastuzumab and AT-101, we have shown that combination significantly correlated with the block of the PI3K/AKT pathway, but not the ERK signal pathway in both breast cancer cells. The PI3K/AKT signaling pathway has a central role in cellular processes critical for tumor progression such as cell growth, survival and motility [4,15]. Consequently, this pathway represents attractive targets for cancer treatment. The PI3K pathway is frequently over activated in breast cancers by several mechanisms including mutations and amplification in key components of this pathway, such as PIK3CA, PIK3R1 and AKT, or loss of PTEN [3,4]. These amplifications are also found in about one-quarter of HER2-positive tumors [4].

The relationship between the PI3K mutation status of patients and clinical outcomes and resistance to some therapies is being studied. In one study, PI3K mutation and PTEN deletion responded less efficiently to trastuzumab suggesting that PI3K mutations that activate this pathway are a mechanism of resistance to anti-HER2 agents [16]. Moreover, the fact that trastuzumab is ineffective in downregulating PI3K/mTOR signaling in trastuzumabresistant models, it is possible to overcome this resistance by the combination treatments [15]. Thus, combining trastuzumab with AT-101, a combination that has shown significant, synergistic, antiproliferative and apoptotic activity against HER2-positive breast cancer cells, might provide a new treatment strategy for HER2-positive breast cancer.

ERK is an MAPK family protein that plays a vital role in the proliferation, survival and differentiation of many tumors $[17,18]$. We investigated the role of ERK in combination-treatment-induced synergistic effects, and demonstrated that there was a significant decrease in the cell viability of combination-treated cells. Based on these data, we suggest that the ERK pathway is not involved in combination-induced cytotoxicity.

We performed combination treatment to induce apoptosis in human breast cancer cells, besides reducing the therapeutic doses of trastuzumab, because tumors arise from an increase in cell number due to a disruption of 
(A)

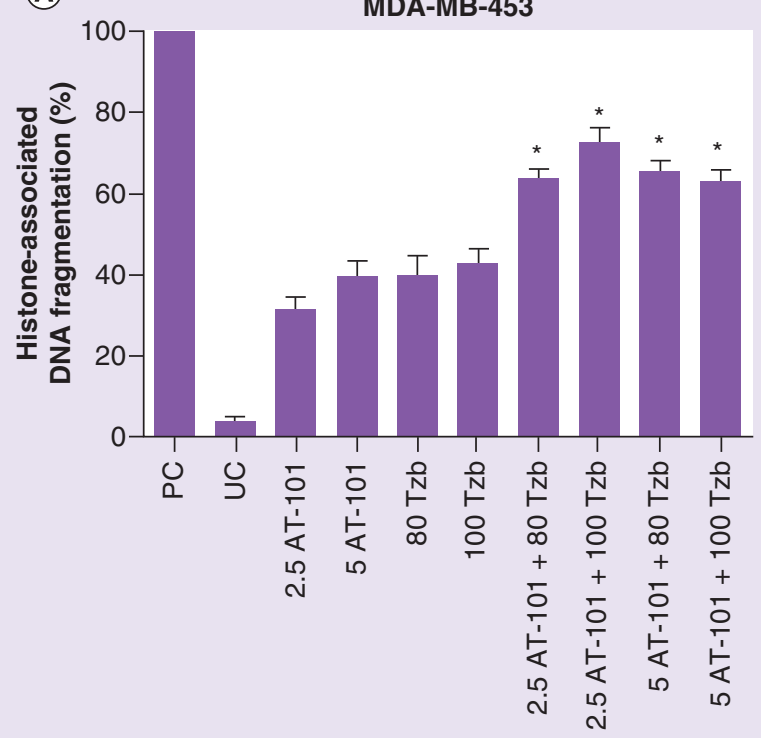

AT-101 $(\mu \mathrm{M})$, trastuzumab $(\mu \mathrm{g} / \mathrm{ml}), 72 \mathrm{~h}$

(C)

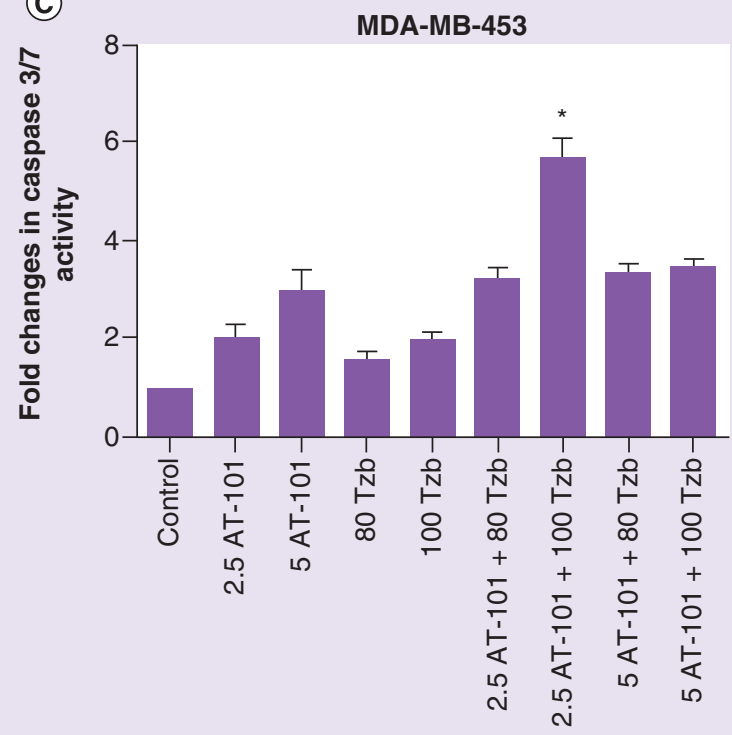

AT-101 $(\mu \mathrm{M})$, trastuzumab $(\mu \mathrm{g} / \mathrm{ml}), 72 \mathrm{~h}$
(B)

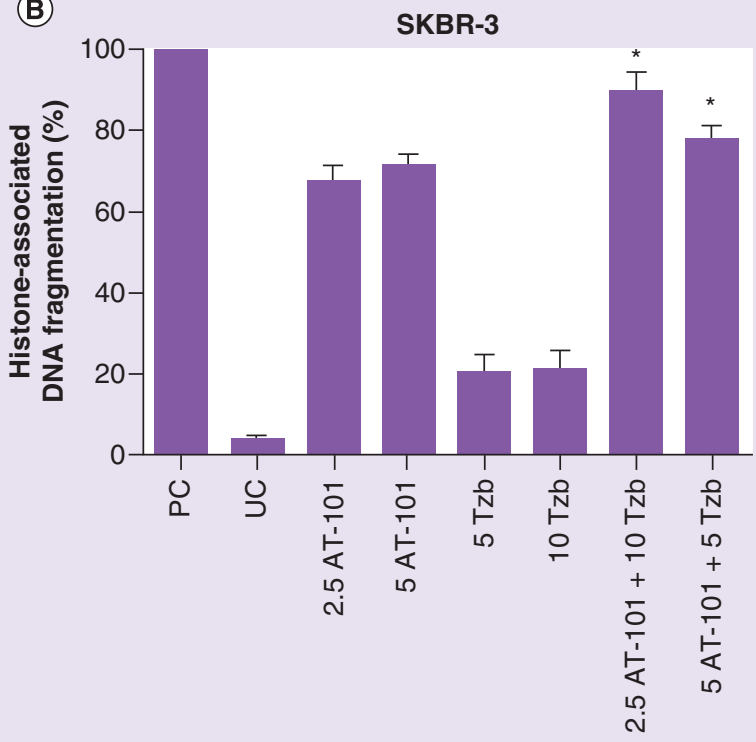

AT-101 $(\mu \mathrm{M})$, trastuzumab $(\mu \mathrm{g} / \mathrm{ml}), 72 \mathrm{~h}$

(D)

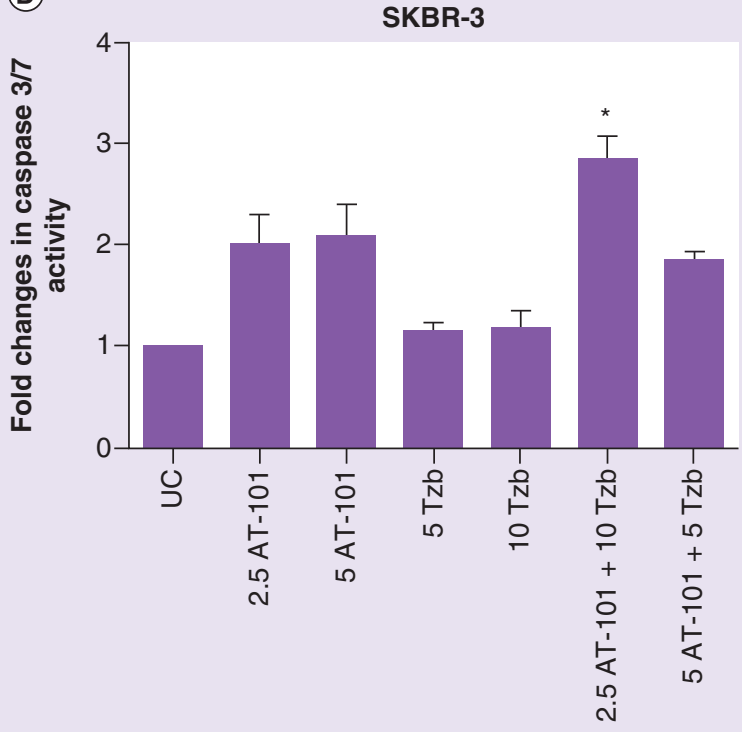

AT-101 $(\mu \mathrm{M})$, trastuzumab $(\mu \mathrm{g} / \mathrm{ml}), 72 \mathrm{~h}$

Figure 5. Effects of trastuzumab/AT-101 combination treatment on (A \& B) DNA fragmentation and (C \& D) caspase 3/7 activity in MDA-MB-453 and SKBR-3 cells at $72 \mathrm{~h} .{ }^{*} \mathrm{p}<0.05$; significant as compared with single drugs.

PC: Positive control; UC: Untreated control.

the balance between cell proliferation and cell death, and also deficiencies in the induction of apoptosis. DNA fragmentation analysis revealed trastuzumab/AT-101 combination-induced apoptotic cell death in both breast cancer cells as compared with single agents.

A strong apoptotic effect is generated through simultaneous activation of the mitochondrial pathway through caspase-8-dependent cleavage of Bid to t-Bid, release of cytochrome $\mathrm{c}$ from the mitochondria, and activation of the extrinsic pathway through caspase-9 [19]. The activation of multiple cell death pathways may help to overcome drug resistance results from single-pathway activation. Therefore, in this study, we performed combination therapy to 
(A)

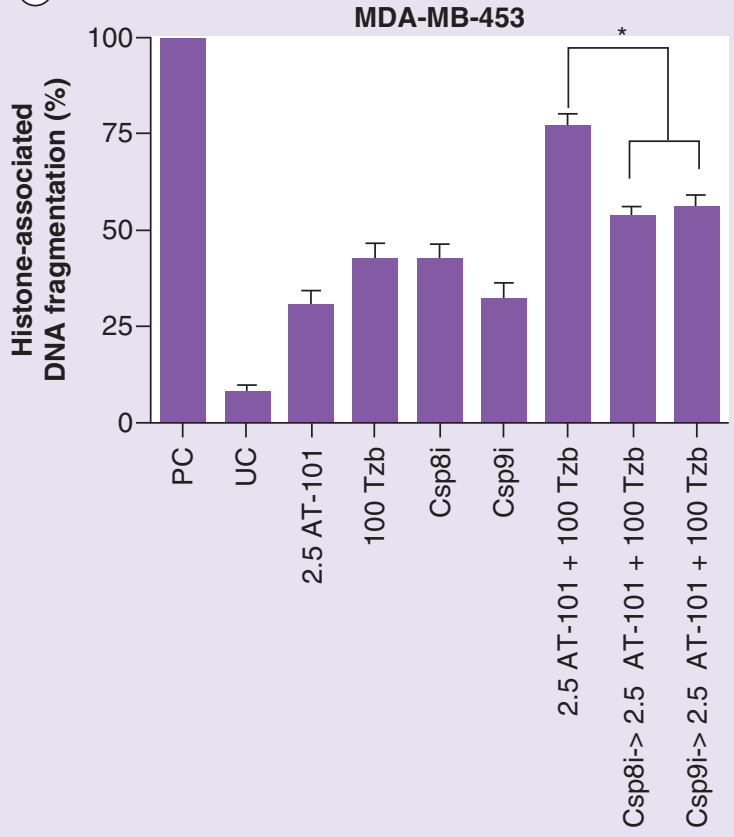

AT-101 $(\mu \mathrm{M})$, trastuzumab $(\mu \mathrm{g} / \mathrm{ml}), 72 \mathrm{~h}$
(B)

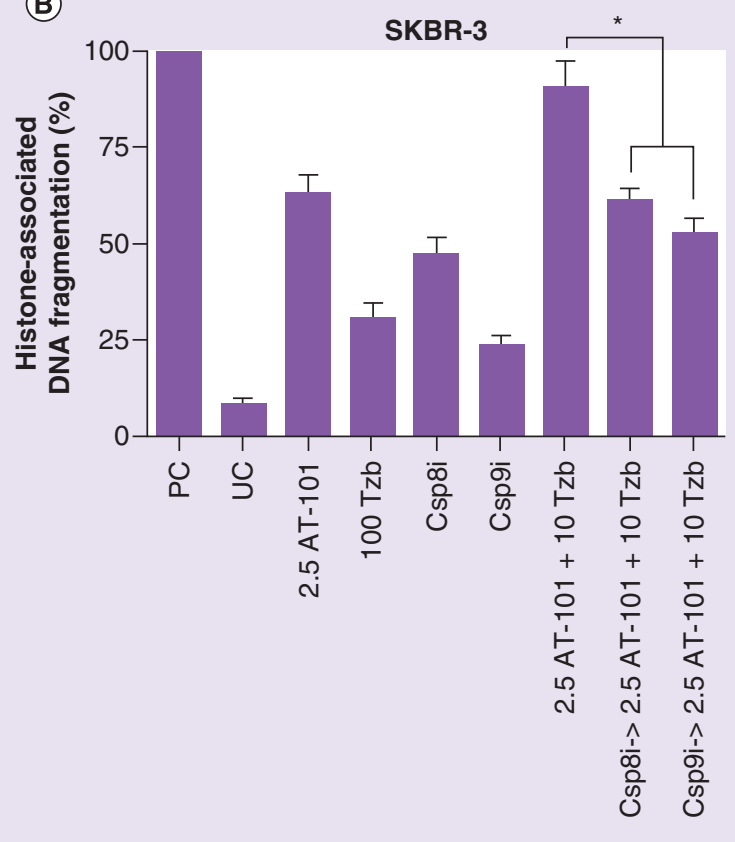

AT-101 $(\mu \mathrm{M})$, trastuzumab $(\mu \mathrm{g} / \mathrm{ml}), 72 \mathrm{~h}$

Figure 6. Inhibition of combination-induced apoptotic cell death by caspase-8 and caspase-9 inhibitors in MDA-MB-453 and SKBR-3 cells. (A \& B) Cells were first incubated with caspase-8 or caspase-p inhibitor for $4 \mathrm{~h}$. Subsequently, drugs were added for $72 \mathrm{~h}$ and a DNA fragmentation analysis was performed.

${ }^{*} \mathrm{p}<0.05$; $\mathrm{p}<0.05$; significant as compared with single drugs.

PC: Positive control; UC: Untreated control.

target multiple apoptotic pathways. Inhibitor experiments showed that trastuzumab, in combination with AT-101, induced both intrinsic and extrinsic apoptotic cell death in HER2-positive breast cancer cells indicating a strong apoptotic effect. Together, caspase- 8 and caspase- 9 activation cause downstream activation of effectors caspase- 3 and caspase-7, which leads to apoptosis. We have also shown that this induction of apoptosis was through a caspase-dependent pathway, which was confirmed by the caspase $3 / 7$ activity assay.

The data presented here provide that trastuzumab in combination with AT-101 induced synergistic cytotoxicity in SKBR3 and MDA-MB-453 HER2-positive breast cancer cell lines. As demonstrated in MCF-10A cells, this synergistic combination may diminish both AT-101 and trastuzumab doses and reduce the toxic effects of these drugs. Moreover, we have shown that combination treatment is a strong inducer of both apoptotic pathways in breast cancer cells. Drugs or combination treatments that trigger apoptosis are good candidates for cancer treatment, and also very ideal for overcoming drug resistance mechanisms in cancer cells. Taken together, the trastuzumab/AT-101 combination may be a good candidate for patients with trastuzumab-resistant HER2-positive breast cancer, and inhibition of the PI3K/AKT pathway may be one of the underlying mechanisms of activity for this combination. In the future, we will expect Trastuzumab / AT-101 combination to be used in animal experiments for years to come. This combination is technologically can convert to a drug form for HER2-positive patients.

There are our some limitations to our study. For example, we know that the MCF-10 breast cell line, which we used as the negative control, is immortalized. The fact that only one normal breast cell line was used in this study is a limitation. However, it does not affect the combination success of trastuzumab and AT-101. In future experiments, it is planned to expand the study by using both more normal cell lines and more HER $2+$ cell lines. Images of the morphology of cells were not able to added. This is another of our study's limitations. This limitation is planned as one of the aims of future research. 


\section{Summary points}

- The HER2 oncogene is overexpressed in $25-30 \%$ of all breast cancer cases and over expression of this oncogene is closely related to shortened disease-free survival. Treatment of these cancers is based on targeted drugs or combinations.

- Trastuzumab is a monoclonal targeted agent against HER2. The most important problem of trastuzumab is drug resistance, which may be primary or secondary. Thus, novel treatment options are required to break resistance mechanisms.

- AT-101(-/-Gossypol) is a polyphenolic compound having potent antiproliferative and apoptotic effects in various cancers. AT-101 also has been studied in breast cancer cell lines and demonstrated apoptotic effect of drug.

- In this cell culture study, two breast cancer cell lines (MDA-MB453 and SKBR-3), in which HER-2 is overexpressed, were used and MCF-10A was used as a control cell line. The drugs were applied to these cell lines alone or in combination and the effects of the drugs on the cells were investigated.

- Trastuzumab/AT101 combination showed a synergistic cytotoxic and apoptotic effect on both HER-2 positive breast cancer cell lines but not on control cells.

- Two pathways were studied that cause this effect. Combination treatment induced cytotoxicity via inhibiting $\mathrm{PI3K} / \mathrm{AKT}$ but not the MAPK/ERK pathway.

- Trastuzumab/AT-101 combination may be a good candidate for patients with trastuzumab-resistant HER-2-positive breast cancer.

Author contributions

G Bulut supervised the development of the study and wrote the manuscript. H Atmaca carried out the viability and apoptosis experiments, wrote the manuscript and carried out the western blot analysis and statistics. B Karaca supervised the manuscript preparation and supplied financial resources. All authors take full responsibility for the content of the final paper.

\section{Acknowledgments}

R Uslu, who is the Department Chair of the authors' institution, supported the project.

\section{Financial \& competing interests disclosure}

This project was supported by The Scientific and Technological Research Council of Turkey (TUBITAK, Project Number 113S055). The authors have no other relevant affiliations or financial involvement with any organization or entity with a financial interest in or financial conflict with the subject matter or materials discussed in the manuscript apart from those disclosed.

No writing assistance was utilized in the production of this manuscript.

Availability of data \& material

All data generated or analyzed during this study are included in this published article.

Ethical conduct of research

The authors state that they have obtained appropriate institutional review board approval (Ege University Ethics Committee 11$1 / 45$ ) or have followed the principles outlined in the Declaration of Helsinki for all human or animal experimental investigations.

Conflict of interest

The authors declare that there is no conflict of interest.

\section{Open access}

This work is licensed under the Attribution-NonCommercial-NoDerivatives 4.0 Unported License. To view a copy of this license, visit http://creativecommons.org/licenses/by-nc-nd/4.0/

\section{References}

Papers of special note have been highlighted as: $\bullet$ of interest; $\bullet \bullet$ of considerable interest

1. Torre LA, Bray F, Siegel RL et al. Global cancer statistics. CA Cancer J. Clin. 65(2), 87-108 (2015).

2. Mayer IA, Arteaga CL. The PI3K/AKT pathway as a target for cancer treatment. Annu. Rev. Med. 67, 11-28 (2015).

3. Baselga J. Targeting the phosphoinositide-3 (PI3) kinase pathway in breast cancer. Oncologist 16(Suppl. 1), 12-19 (2011).

4. Courtney KD, Corcoran RB, Engelman JA. The PI3K pathway as drug target in human cancer. J. Clin. Oncol. 28(6), 1075-1083 (2010). 
5. Nahta R, Esteva FJ. HER2 therapy: molecular mechanisms of trastuzumab resistance. Breast Cancer Res. 8(6), 215 (2006).

- Mechanisms of trastuzumab resistance leads to the investigation of the PI3K pathway.

6. Paulus A, Chitta K, Akhtar S et al. AT-101 downregulates Bcl-2 and MCL1 and potentiates the cytotoxic effects of lenalidomide and dexamethasone in preclinical models of multiple myeloma and Waldenström macroglobulinaemia. Br. J. Haematol. 164(3), 352-365 (2014).

-• Preclinical study demonstrates antitumor responses of AT-101 which is BH-3 mimetic agent in multiple myeloma.

7. Oliver CL, Bauer JA, Wolter KG et al. In vitro effects of the BH3 mimetic, (-)-gossypol, on head and neck squamous cell carcinoma cells. Clin. Cancer Res. 10(22), 7757-7763 (2004).

- Preclinical study demonstrates antitumor responses of AT-101 which is BH-3 mimetic agent in head and neck squamous cell carcinoma cells.

8. Mohammad RM, Wang S, Aboukameel A et al. Preclinical studies of a nonpeptidic small-molecule inhibitor of Bcl-2 and Bcl-X(L) [(-)-gossypol] against diffuse large cell lymphoma. Mol. Cancer Ther. 4(1), 13-21 (2005).

9. Liu G, Kelly WK, Wilding G et al. An open-label, multicenter, Phase I/II study of single-agent AT-101 in men with castrate-resistant prostate cancer. Clin. Cancer Res. 15, 3172-3176 (2009).

10. Van Poznak C, Seidman AD, Reidenberg MM et al. Oral gossypol in the treatment of patients with refractory metastatic breast cancer: a phase I/II clinical trial. Breast Cancer Res. Treat. 66(3), 239-248 (2001).

11. Baggstrom MQ, Qi Y, Koczywas M et al. A Phase II study of AT-101 (Gossypol) in chemotherapy-sensitive recurrent extensive-stage small cell lung cancer. J. Thorac. Oncol. 6, 1757-1760 (2011).

-• A Phase II study and AT-101 demonstrate the antitumor effect in extensive-stage of the small cell lung cancer.

12. Masood A, Chitta K, Paulus A et al. Downregulation of BCL2 by AT-101 enhances the antileukaemic effect of lenalidomide both by an immune dependent and independent manner. Br. J. Haematol. 157, 59-66 (2012).

13. Chou TC, Talalay P. Quantitative analysis of dose-effect relationships: the combined effects of multiple drugs or enzyme inhibitors. Adv. Enzyme Regul. 22, 27-55 (1984).

14. Narayan M, Wilken JA, Harris LN et al. Trastuzumab-induced HER reprogramming in 'resistant' breast carcinoma cells. Cancer Res. 69(6), 2191-2004 (2009)

15. Akinleye A, Avvaru P, Furqan M, Song Y, Liu D. Phosphatidylinositol 3-kinase (PI3K) inhibitors as cancer therapeutics. J. Hematol. Oncol. 6(1), 88 (2013).

16. O’Brien NA, McDonald K, Tong L et al. Targeting PI3K/mTOR overcomes resistance to HER2-targeted therapy independent of feedback activation of AKT. Clin. Cancer Res. 20(13), 3507-3520 (2011).

-. PI3K/AKT pathway demonstrates an important role in trastuzumab resistance.

17. Rovida E, Stecca B. Mitogen-activated protein kinases and Hedgehog-GLI signaling in cancer: a crosstalk providing therapeutic opportunities? Cancer Biol. 35, 154-167 (2015).

18. Samatar AA, Poulikakos PI. Targeting RAS-ERK signaling in cancer: promises and challenges. Nat. Rev. Drug Discov. 13(12), 928-942 (2014).

19. Ouyang L, Shi Z, Zhao S et al. Programmed cell death pathways in cancer: a review of apoptosis, autophagy and programmed necrosis. Cell Prolif. 45(6), 487-498 (2012). 
